\title{
What Do You Get If You Cross a Phase Object Approximation with a Dynamically Scattering Sample?
}

L Clark $^{1}$, Colum O’Leary ${ }^{1}$, GT Martinez ${ }^{1}$, TC Petersen ${ }^{2}$, Scott Findlay $^{3}$ and Peter Nellist ${ }^{1}$

${ }^{1}$ University of Oxford, Oxford, England, United Kingdom, ${ }^{2}$ Monash University, Melbourne, Victoria, Australia, ${ }^{3}$ Monash University, Clayton, Victoria, Australia

The recent developments in fast-readout electron detectors have opened new doors on the imaging and image analysis we can perform in the STEM. 4D-STEM algorithms are enabling higher-contrast imaging [1] at lower dose [2] of challenging and technologically important materials [3].

Inherent to many of our imaging algorithms, however, are approximations which do not always hold for the actual specimens of interest in the study of real materials-science problems. The (weak) phase object approximation is commonly used but can break down for both long-range fields [4] and on-axis crystals [5], giving potentially inaccurate results. The full-width of an electron probe in the sample plane can be significantly wider than double the full-width at half max - increasing the probability of phase curvature under the beam and thus breaking the linear phase gradient approximation in the conventionally truncated Taylor expansion of the phase. Channelling effects can become significant at surprisingly low specimen thicknesses [6] in ways which cannot be described simply with a single phase-object model (although, they can in some cases be mitigated by precession imaging [7]).

The breakdown of our conventional models in sample systems we wish to understand further motivates the present work: a quantitative study of atomic-resolution 4D-STEM imaging methods in cases where dynamical scattering is non-negligible. To build a full understanding of how dynamical scattering presents in 4D-STEM datasets, we start with a simulation of a thickness series of a perfect, on-axis crystal. These datasets can then be analysed with a range of STEM imaging algorithms (see Figure 1 for a subset of these).

At certain depths in this thickness series, the atomic peaks are replaced by unphysical caldera profiles (this is seen, for example in the upper row of Figure 1 at 25 unit cells thick). As we are working from ideal, simulated data, we can ascribe these features to dynamical scattering, and then focus on a more detailed study of the data points leading to these artefacts by analysing the ronchigrams associated with the relevant pixel positions. In the case of single-side band ptychography analysis [8], we can further study the data in spatial-frequency space (see Figure 2 for an example of this) associated with that pixel position in order to tease out how these artefacts associated with dynamical scattering form from features in the dataset.

We note that these caldera profiles appear at different depths for the various imaging methods representative of the fact that the images resulting from the algorithms have sampled and weighted the 4D data set in different ways - suggesting a route forwards to mitigate these features for a more easily interpretable 4D-STEM image in the presence of dynamical scattering [9]. 


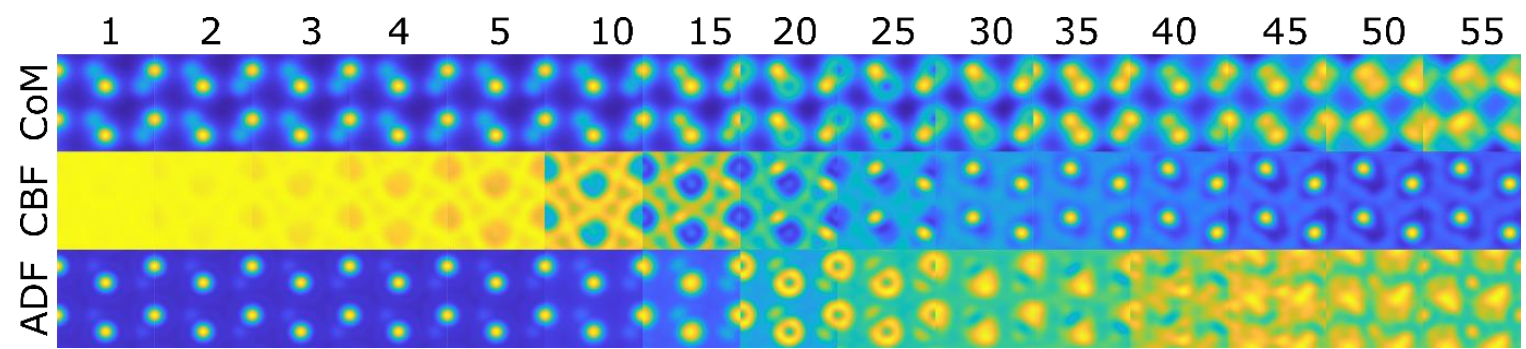

Figure 1. Annular dark field (ADF), central bright field $(\mathrm{CBF})$ and integrated centre of mass $(\mathrm{CoM})$ images over a simulated thickness series of $\mathrm{GaN}$

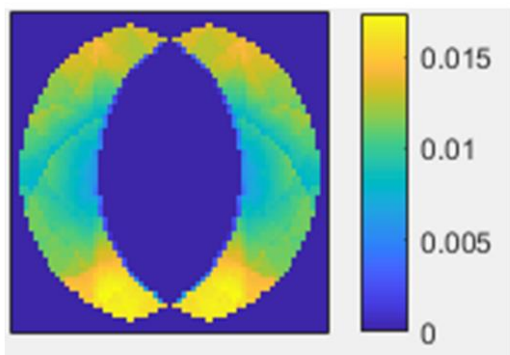

Figure 2. The intensity of the double overlap regions (in reciprocal space) of one selected spatial frequency from 30 unit cells of GaN. Note the non-uniform intensity within the segments - a result of dynamical scattering.

\section{References}

[1] Pennycook, Timothy J., et al. "High dose efficiency atomic resolution imaging via electron ptychography." Ultramicroscopy 196 (2019): 131-135.

[2] O'Leary, Colum M., et al. "Contrast Transfer and Noise Minimization in Electron Ptychography." Microscopy and Microanalysis 25.S2 (2019): 64-65.

[3] Lozano, Juan G., et al. "Low-dose aberration-free imaging of Li-rich cathode materials at various states of charge using electron ptychography." Nano letters 18.11 (2018): 6850-6855.

[4] Clark, L., et al. "Probing the limits of the rigid-intensity-shift model in differential-phase-contrast scanning transmission electron microscopy." Physical Review A 97.4 (2018): 043843.

[5] Kirkland, Earl J. Advanced computing in electron microscopy. Springer Science \& Business Media, 2010.

[6] Aveyard, Richard, et al. "Modeling nanoscale inhomogeneities for quantitative HAADF STEM imaging." Physical review letters 113.7 (2014): 075501.

[7] Mawson, T., et al. "Suppressing dynamical diffraction artefacts in differential phase contrast scanning transmission electron microscopy of long-range electromagnetic fields via precession." arXiv preprint arXiv:2002.01595 (2020).

[8] Pennycook, Timothy J., et al. "Efficient phase contrast imaging in STEM using a pixelated detector. Part 1: Experimental demonstration at atomic resolution." Ultramicroscopy 151 (2015): 160-167

[9] Acknowledgements: The authors acknowledge funding from ESTEEM3 under the Horizon2020 programme. SDF acknowledges support from the Australian Research Council Discovery Projects funding scheme (Project DP160102338). 